\title{
ON POWER DRAZIN NORMAL AND DRAZIN QUASI-NORMAL HILBERT SPACE OPERATORS
}

\author{
B. P. DugGal And I. H. KIM
}

Abstract. A Drazin invertible Hilbert space operator $T \in B(\mathscr{H})$, with Drazin inverse $T_{d}$, is $(n, m)$-power D-normal, $T \in[(n, m) D N]$, if $\left[T_{d}^{n}, T^{* m}\right]=T_{d}^{n} T^{* m}-T^{* m} T_{d}^{n}=0 ; T$ is $(n, m)$ power D-quasinormal, $T \in[(n, m) D Q N]$, if $\left[T_{d}^{n}, T^{* m} T\right]=0$. Operators $T \in[(n, m) D N]$ have a representation $T=T_{1} \oplus T_{0}$, where $T_{1}$ is similar to an invertible normal operator and $T_{0}$ is nilpotent. Using this representation, we have a keener look at the structure of $[(n, m) D N]$ and $[(n, m) D Q N]$ operators. It is seen that $T \in[(n, m) D N]$ if and only if $T \in[(n, m) D Q N]$, and if $[T, X]=0$ for some operators $X \in B(\mathscr{H})$ and $T \in[(1,1) D N]$, then $\left[T_{d}^{*}, X\right]=0$. Given simply polar operators $S, T \in[(1,1) D N]$ and an operator $A=\left(\begin{array}{cc}T & C \\ 0 & S\end{array}\right) \in B(\mathscr{H} \oplus \mathscr{H}), A \in[(1,1) D N]$ if and only if $C$ has a representation $C=0 \oplus C_{22}$.

Mathematics subject classification (2010): 47A15, 47B15, 47B20.

Keywords and phrases: Drazin invertible operator, structure of $[(n, m) D N]$ operators, $n$-th root of normal operator, commutativity theorem.

\section{REFERENCES}

[1] P. AiEnA, Fredholm and Local Spectral Theory II with Applications to Weyl-type Theorems, Lecture Notes in Mathematics 2235, Springer (2018).

[2] M. DANA AND R. Yousefi, On the classes of D-normal and D-quasinormal operators, Operators and Matrices 12(2)(2018), 465-487.

[3] D.S. Djordjevic And V. Rakocevic, Lectures on Generalized Inverse, Faculty of Sciences and Mathematics, University of Nis, 2008.

[4] B.P. DugGal, Finite intertwinings and subscalarity, Operators and Matrices 4(2010), 257-271.

[5] B.P. DugGaL, On characterising contractions with $C_{10}$ pure part, Integral Equat. Oper. Th. 27(1997), 314-323.

[6] M.R. EMBRY, nth roots of normal operators, Proc. Amer. Math. Soc. 19(1968), 63-68.

[7] J. ESCHMEIER AND M. PUTINER, Bishop's property $(\beta)$ and rich extensions of linear operators, Indiana Univ. Math. J. 37(1988), 325-348.

[8] F. Gilfeather, Operator valued roots of abelian analytic functions, Pac. J. Math. 55(1974), 127148.

[9] P.R. Halmos, A Hilbert Space Problem Book, Second Edition (1982), Springer-Verlag, New York Heidelberg - Berlin.

[10] C.S. Kubrusly, Hilbert Space Operators - A Problem Solving Approach, Birkhäuser, 2003.

[11] K.B. Laursen and M.N. Neumann, Introduction to Local Spectral Theory, Clarendon, Oxford 2000.

[12] M. Radjavi And P. Rosenthal, On roots of normal operators, J. Math. Anal. Appl. 34(2)(2013), 653-665.

[13] O.A.M. Sid Ahmed And O.B. Sid Ahmed, On the classes $(n, m)$ - power D-normal and $(n-m)$ power D-quasinormal operators, Operators and Matrices 13(3)(2019), 705-732.

[14] J.G. Stampfli, Roots of scalar operators, Proc. Amer. Math. Soc. 13(1962), 796-798.

[15] A.E. TAYLOR AND D.C. LAY, Introduction to Functional Analysis, Wily, New York, 1980. 